\title{
Clinical Significance of Germline Telomere Length and Associated Genetic Factors in Patients with Neuroblastoma
}

\author{
Ki Woong Sung ( $\sim$ kwsped@skku.edu ) \\ Samsung Medical Center, Sungkyunkwan University School of Medicine
} Joon Seol Bae

Samsung Medical Center

Ji Won Lee

Samsung Medical Center, Sungkyunkwan University School of Medicine

Je-Gun Joung

Cha University

\section{Hee Won Cho}

Samsung Medical Center, Sungkyunkwan University School of Medicine

Hee Young Ju

Samsung Medical Center, Sungkyunkwan University School of Medicine

Keon Hee Yoo

Samsung Medical Center, Sungkyunkwan University School of Medicine

Hong Hoe Koo

Samsung Medical Center, Sungkyunkwan University School of Medicine

\section{Research Article}

Keywords: telomere length, neuroblastoma, genetic marker, survival duration, genome-wide association study

Posted Date: March 3rd, 2022

DOI: https://doi.org/10.21203/rs.3.rs-1360590/v1

License: (c) (i) This work is licensed under a Creative Commons Attribution 4.0 International License. Read Full License 


\section{Abstract}

Background: Studies investigating the relationship between germline telomere length and the clinical characteristics of tumors are very limited. This study evaluated the relationship between germline telomere length and the clinical characteristics of neuroblastoma. In addition, a genome-wide association study (GWAS) was performed to investigate the genetic factors associated with telomere length.

Methods: The telomere length of peripheral blood mononuclear cells from 186 patients with neuroblastoma was measured by quantitative PCR. The association between germline telomere length and clinical characteristics, including long-term survival, was investigated. For the GWAS, genotyping was performed with a high-density Illumina bead chip. After strict quality control checks of the samples, an association analysis was conducted.

Results: An extra-abdominal primary site, lower stage (stage 1/2), MYCN non-amplified tumor, and being in the low-risk group were associated with longer telomere lengths $(P=0.015,0.007,0.046$ and 0.042 , respectively). Patients with longer third telomeres had longer event-free survival than other patients $(P=0.032)$. In the GWAS, rs2492082, located in the 3 'UTR of the HIVEP3 gene, showed the highest association with telomere length $(P=1.7 \mathrm{E}-06)$. Patients with long telomeres had a recessive allele A. A gene-based analysis using the VEGAS2 algorithm showed that the CNTN4 gene had the most significant association with telomere length $(P=1.0 \mathrm{E}-06)$. Patients with the $\mathrm{CC}$ homozygote in the rs71311728 marker within the CNTN4 gene had a longer telomere length than those with the TC heterozygote. A gene ontology analysis showed that genetically susceptible genes associated with telomere length were mainly distributed in neurite morphogenesis and neuron development.

Conclusions: Longer germline telomere length is associated with favorable prognostic factors at diagnosis and eventually better event-free survival in patients with neuroblastoma. In addition, the GWAS demonstrated that genetic markers and genes related to telomere length are associated with neurite morphogenesis and neuron development. A further study with a larger cohort of patients and functional studies are needed.

\section{Introduction}

Telomeres are DNA-protein complexes at the ends of chromosomes that determine the lifespans of cells. As a repeat sequence (TTAGGC) present at the end of the genome, telomeres are known to play a role in preventing damage to the genome ${ }^{1}$. They protect chromosomes from end-to-end fusion ${ }^{2}$ and are involved in a variety of other functions, including cell death, cell senescence, abnormal cell proliferation, and separation during meiosis ${ }^{3,4}$. Telomere length has been reported to be inherited and has been found to vary between individuals of the same age ${ }^{5}$. Telomere length decreases when cells divide, and as telomere length decreases, cells age and die. Thus, telomere length has a profound relationship with age-related diseases and cancer ${ }^{6-8}$. Aging caused by telomere shortening differs from individual to individual and is known to be affected by environmental ${ }^{9}$ and genetic differences ${ }^{10}$.

Many genetic epidemiologic studies have been reported the results of analysis of the association between telomere length and cancer risk ${ }^{11-14}$. A significant association of short telomere length with cancer risk in peripheral blood leucocytes, lung cancer, bladder, head and neck, lung, and renal cell cancers, and breast cancer has been reported 11,12,15-17. Functional studies using mouse model have shown that shorter telomere lengths are associated with an increased risk of cancers such as epithelial caner, prostate cancer ${ }^{12,18-20}$. A shorter telomere length may promote cell aging and inhibit cancer progression. However, when the critical telomere length is reached, it would cause genomic instability expanding and malignant transformation potential through the fusion bridge breakage cycle ${ }^{21}$. Conversely, conflicting results have also been reported, suggesting that longer telomere lengths increase cancer risk ${ }^{22-25}$. A genetic studies that measures the telomere length in various type of cancer for various populations and analyzes the association with telomere length with clinical characteristics of cancer patients is necessary to obtain more accurate conclusion. In particular, in the case of neuroblastoma, attempts to analyze the association between clinical features by measuring the telomere length of a cancer patient are very insufficient. 
Telomere length, which is associated with chromosomal instability and thus the degree of cell protection from external stimuli, might also be associated with the clinical characteristics of tumors, including treatment outcomes. However, studies investigating the relationship between germline telomere length and the clinical characteristics of tumors are very limited. This study evaluated the relationship between germline telomere length and the clinical characteristics of neuroblastoma. In addition, a genome-wide association study (GWAS) was performed to investigate the genetic factors associated with telomere length.

\section{Methods}

\section{Patients}

A total of 186 patients who were diagnosed with neuroblastoma between May 2007 and July 2016 and had peripheral blood samples already cryopreserved at the Samsung Medical Center Biobank were enrolled in this study. Medical records were reviewed to obtain detailed clinical and biological data, such as the clinical presentation at diagnosis, tumor biology (including MYCN amplification status), tumor histology using the International Neuroblastoma Pathology Classification, and survival.

This study was approved by the Institutional Review Board of Samsung Medical Center (IRB No. SMC 2015-11-053-035) and conducted according to the Declaration of Helsinki. All patients signed an informed consent before enrolled in this research and all methods were performed in accordance with the relevant guidelines and regulations.

\section{Genome-wide genotyping}

Genomic DNA was extracted from the collected blood using a Wizard Genomic DNA Purification Kit (Promega, WI, USA). DNA quantification was measured by fluorescence using Qubit equipment, and DNA integrity was checked using TapeStation equipment (Agilent, Santa Clara, USA). 200 ng of DNA that passed quality control (QC) was used for the Illumina Infinium assay. The chip used in this study is an Illumina Infinium Exome-24 BeadChip array (Illumina, San Diego, USA) containing 547,644 markers. The samples were processed according to the Illumina Infinium assay manual. Each sample was wholegenome amplified, fragmented, precipitated, and resuspended in an appropriate hybridization buffer. The denatured samples were then hybridized on a prepared GSA BeadChip for a minimum of $16 \mathrm{~h}$ at $48^{\circ} \mathrm{C}$. Following hybridization, the bead chips were processed for the single-base extension reaction, staining, and imaging on an Illumina iScan system. The normalized bead intensity data obtained for each sample were uploaded to GenomeStudio software (Illumina, San Diego, USA), which converted the fluorescent intensities into single nucleotide polymorphism (SNP) genotypes. Sample quality was checked using a sample call rate of $>95 \%$. In GenomeStudio (Illumina, San Diego, USA), the cluster quality was measured using GenTrain scores, and then high-quality markers (>0.7) were used.

\section{Telomere length measurement}

Quantitative real-time polymerase chain reaction (qPCR) was used to measure telomere length ${ }^{26}$. The relative telomere lengths were measured as the ratio of the number of telomere $(T)$ repeat copies to the number of single copy gene (S) copies ( $T: S$ ratio) in a given sample. Telomere length was calculated as the ratio between the length of the test DNA and the pooled reference DNA. Three replicates were performed on patient samples to calculate the average T:S ratio.

\section{Statistics}

The clinical variables are summarized using mean \pm standard deviation or median (range), as appropriate (Table 1). In HelixTree (Golden Helix Inc., Bozeman, MT, USA), marker filtering was performed based on the following criteria: (1) call rate > 0.85 , (2) minor allele frequency $>0.05$, and Hardy Weinberg equilibrium $P$ value $>0.001$. High-quality markers with values above those QC criteria were used in the following analyses. For the GWAS, genotype distributions were compared using multivariable regression analyses in HelixTree software (Golden Helix Inc., Bozeman, MT, USA). The gene-based analysis was performed using the VEGAS2 algorithm (https://vegas2.qimrberghofer.edu.au/). In the VEGAS2 options, we choose 1000G 
Asians for SNPs and all Asians for sub-population. The analysis was carried out for three groups: all and $10 \%$ and $20 \%$ extreme groups. A gene pathway analysis was done using VEGAS2. The event-free survival (EFS) and overall survival (OS) rates were estimated using the Kaplan-Meier method, and differences in survival curves were compared using the log-rank test. A multivariate analysis for EFS was performed using a Cox regression analysis. Clinical characteristics were compared between two groups using the Pearson chi-square test or Fisher's exact test for categorical variables and the $t$ test or KruskalWallis rank-sum test for continuous variables. $P$ values $<0.05$ were considered significant. 
Table 1

Telomere length according to clinical characteristics

\begin{tabular}{|c|c|c|c|}
\hline Clinical Characteristics & No. (\%) & Median (range) & $P$-value \\
\hline Sex & & & 0.820 \\
\hline Male & $99(53.2)$ & $17.51(8.28-29.36)$ & \\
\hline Female & $87(46.8)$ & $17.18(8.41-26.73)$ & \\
\hline Age & & & 0.113 \\
\hline$<1.5$ years & $73(39.2)$ & $18.05(8.41-29.36)$ & \\
\hline$>1.5$ years & $113(60.8)$ & $17.22(8.28-25.17)$ & \\
\hline Primary site & & & 0.015 \\
\hline Abdomen & $141(75.8)$ & $17.18(8.41-27.46)$ & \\
\hline Others & $45(24.2)$ & $18.78(8.28-29.36)$ & \\
\hline Stage & & & 0.007 \\
\hline 1,2 & $50(26.9)$ & $18.44(8.28-29.36)$ & \\
\hline $3,4,4 S$ & $136(73.1)$ & $17.11(8.41-25.70)$ & \\
\hline Differentiation $(\mathrm{N}=166)$ & & & 0.891 \\
\hline GNB & $39(23.5)$ & $18.00(8.28-25.17)$ & \\
\hline Differentiating & $37(22.3)$ & $17.85(9.93-25.10)$ & \\
\hline PD/UD & $90(54.2)$ & $17.65(8.48-29.36)$ & \\
\hline MYCN amplification $(N=183)$ & & & 0.046 \\
\hline Absent & $155(84.7)$ & $17.85(8.28-29.36)$ & \\
\hline Present & $28(15.3)$ & $16.20(8.48-24.73)$ & \\
\hline 1p deletion $(\mathrm{N}=99)$ & & & 0.856 \\
\hline Absent & $86(86.9)$ & $18.41(11.07-27.46)$ & \\
\hline Present & $13(13.1)$ & $17.80(15.20-25.17)$ & \\
\hline 11q deletion $(\mathrm{N}=98)$ & & & 0.893 \\
\hline Absent & $73(74.5)$ & $18.57(11.07-27.46)$ & \\
\hline Present & $25(25.5)$ & $18.10(12.72-25.70)$ & \\
\hline $17 q$ gain $(\mathrm{N}=97)$ & & & 0.300 \\
\hline Absent & $66(68.0)$ & $18.41(11.07-27.46)$ & \\
\hline Present & $31(32.0)$ & $18.46(15.41-25.70)$ & \\
\hline Risk group & & & 0.042 \\
\hline
\end{tabular}

Abbreviations: Ganglioneuroblastoma (GNB), poorly differentiated (PD), undifferentiated (UD)

Stage 1,2, and 4S tumors were stratified into the low-risk group if MYCN was not amplified, whereas stage 4 tumors in patients older than 12 months (until 2008) or 18 months (since 2009) or any tumors with amplified MYCN were classified as the high-risk group. The intermediate-risk group includes all other tumors not mentioned above. 


\begin{tabular}{|lcc|}
\hline Clinical Characteristics & No. (\%) & Median (range) \\
\hline Low & $50(26.9)$ & $18.36(8.28-29.36)$ \\
\hline Intermediate & $49(26.3)$ & $17.18(8.41-25.70)$ \\
\hline High & $87(46.8)$ & $17.44(8.28-29.36)$ \\
\hline Abbreviations: Ganglioneuroblastoma (GNB), poorly differentiated (PD), undifferentiated (UD) \\
\hline $\begin{array}{l}\text { Stage 1, 2, and 4S tumors were stratified into the low-risk group if MYCN was not amplified, whereas stage } 4 \text { tumors in } \\
\text { patients older than 12 months (until 2008) or 18 months (since 2009) or any tumors with amplified MYCN were classified } \\
\text { as the high-risk group. The intermediate-risk group includes all other tumors not mentioned above. }\end{array}$ \\
\hline
\end{tabular}

\section{Results}

\section{Clinical characteristics and their association with telomere length}

The clinical characteristics of the 186 patients are given in Table 1. The median age was 2.1 years (range, 0.0-19.3), and 87 (46.8\%) patients were categorized into the high-risk group. In addition, 28 (15.3\%) patients had MYCNamplification. We tested the association between telomere length and ten important clinical characteristics in neuroblastoma: sex, age, primary site, tumor stage, tumor differentiation, MYCN amplification, 1p deletion, 11q deletion, 17q gain, and risk group. We found that an extra-abdominal primary site, lower stage (stage 1/2), MYCN non-amplified tumor, and low-risk group categorization were associated with longer telomere length $(P=0.015,0.007,0.046$, and 0.042 , respectively). Patients with longer third telomeres had longer EFS than other patients $(P=0.032)$, but there was no difference in OS (Fig. 1). Table 2 lists the results of the multivariate analysis for EFS. Age older than 1.5 years (hazard ratio 3.08, $P=0.017$ ), stage 4 (hazard ratio $4.75, P=0.003$ ), and longer third telomere length (hazard ratio $0.037, P=0.046$ ) were independent prognostic factors for EFS.

Table 2

Multivariate analysis for event-free survival (EFS)

\begin{tabular}{|lll|}
\hline Risk Factors & Hazard ratio (range) & $P$-value \\
\hline Age $>1.5$ years & $3.08(1.22-7.78)$ & 0.017 \\
\hline Stage 4 & $4.75(1.70-13.26)$ & 0.003 \\
\hline MYCN amplification & $1.40(0.65-3.03)$ & 0.392 \\
\hline Differentiation & & 0.456 \\
\hline GND & 1.00 & \\
\hline Differentiating & $0.88(0.28-2.85)$ & 0.840 \\
\hline PD/UD & $1.46(0.53-4.00)$ & 0.466 \\
\hline Telomere length & & 0.131 \\
\hline Shorter third & 1.00 & \\
\hline Middle third & $0.71(0.36-1.42)$ & 0.338 \\
\hline Longer third & $0.37(0.14-0.98)$ & 0.046 \\
\hline
\end{tabular}

\section{Genetic association analysis of telomere length}

We tested associations with telomere length in neuroblastoma patients in a multivariable regression analysis using age and sex as covariates. After marker filtering, 248,399 markers were used in the association analysis. We found that telomere length correlated with several significant genes: HIVEP3, LRRTM4, ADGRV1, RAB30, and CHRNA4 (Fig. 2A). The significant 
markers for telomere length are summarized in Table 3. In the GWAS, the most highly associated marker was rs10842679 (P $=4.7 \mathrm{E}-07$ ). The gene nearest rs 10842679 was $B H L H E 41$, which is known as a putative regulator of neuronal differentiation ${ }^{27}$. Interestingly, markers located in the 3'UTR of the HIVEP3 gene showed a strong association with telomere length (Table 3).

The telomere length in the markers tends to increase from the dominant allele to the recessive allele. We analyzed the regional associations of $400 \mathrm{~kb}$ around HIVEP3 on chromosome 1p34.2 (Fig. 2B) and found that the rs2492082 marker had a relatively robust association signal ( $P=1.7$.E-06, Table 3, Fig. 2B). 
Table 3

Single nucleotide polymorphisms associated with telomere length in neuroblastoma patients

\begin{tabular}{|c|c|c|c|c|c|c|c|c|c|c|}
\hline SNP & CHR & BP & Alleles & Gene & Region & MAF & $\mathrm{DD}(\mathrm{TL})$ & $\operatorname{Dd}(T L)$ & $\mathrm{dd}(T L)$ & $\begin{array}{l}P \\
\text { value }\end{array}$ \\
\hline rs10842679 & 12 & 26281858 & $\mathrm{G}>\mathrm{C}$ & & & 0.084 & 155(16.6) & $29(20.1)$ & $1(25.2)$ & $\begin{array}{l}4.7 \mathrm{E}- \\
07\end{array}$ \\
\hline rs10890075 & 1 & 41970768 & $\mathrm{~T}>\mathrm{C}$ & & & 0.395 & $67(15.6)$ & $90(17.7)$ & $28(19.5)$ & $\begin{array}{l}1.2 \mathrm{E}- \\
06\end{array}$ \\
\hline rs11210339 & 1 & 41971212 & $A>G$ & & & 0.392 & $68(15.6)$ & $89(17.7)$ & $28(19.5)$ & $\begin{array}{l}1.7 \mathrm{E}- \\
06\end{array}$ \\
\hline rs2492082 & 1 & 41972198 & $\mathrm{~T}>\mathrm{A}$ & HIVEP3 & UTR3 & 0.392 & $68(15.6)$ & $89(17.7)$ & $28(19.5)$ & $\begin{array}{l}1.7 \mathrm{E}- \\
06\end{array}$ \\
\hline rs1383116 & 12 & 26278079 & $A>C$ & & & 0.081 & 156(16.7) & $28(20.0)$ & $1(25.2)$ & $\begin{array}{l}1.8 \mathrm{E}- \\
06\end{array}$ \\
\hline rs71420970 & 2 & 77012296 & $C>G$ & LRRTM4 & intron & 0.070 & 159(16.7) & $26(20.5)$ & & $\begin{array}{l}3.5 \mathrm{E}- \\
06\end{array}$ \\
\hline rs6679278 & 1 & 70851205 & $G>A$ & & & 0.254 & 106(16.1) & $64(18.5)$ & 15(19.6) & $\begin{array}{l}3.6 \mathrm{E}- \\
06\end{array}$ \\
\hline rs11243436 & 9 & 134449467 & $\mathrm{C}>\mathrm{T}$ & & & 0.100 & 152(16.6) & 29(19.6) & $4(22.3)$ & $\begin{array}{l}3.8 \mathrm{E}- \\
06\end{array}$ \\
\hline rs11243437 & 9 & 134450073 & $C>G$ & & & 0.100 & 152(16.6) & $29(19.6)$ & $4(22.3)$ & $\begin{array}{l}3.8 \mathrm{E}- \\
06\end{array}$ \\
\hline rs12806316 & 11 & 113495279 & $C>A$ & & & 0.414 & $63(15.4)$ & $91(17.9)$ & $31(18.9)$ & $\begin{array}{l}3.9 \mathrm{E}- \\
06\end{array}$ \\
\hline rs2492080 & 1 & 41971867 & $A>C$ & & & 0.395 & $67(15.7)$ & $90(17.7)$ & $28(19.3)$ & $\begin{array}{l}5.3 \mathrm{E}- \\
06\end{array}$ \\
\hline rs35359723 & 2 & 77015092 & $G>A$ & LRRTM4 & intron & 0.065 & 161(16.7) & $24(20.6)$ & & $\begin{array}{l}5.5 \mathrm{E}- \\
06\end{array}$ \\
\hline rs2731765 & 16 & 58276591 & $C>A$ & & & 0.300 & $87(18.2)$ & $85(16.8)$ & 13(12.9) & $\begin{array}{l}6.5 \mathrm{E}- \\
06\end{array}$ \\
\hline rs2257931 & 16 & 58279244 & $A>G$ & & & 0.300 & $87(18.2)$ & $85(16.8)$ & $13(12.9)$ & $\begin{array}{l}6.5 \mathrm{E}- \\
06\end{array}$ \\
\hline rs1969749 & 1 & 41973908 & $\mathrm{~T}>\mathrm{C}$ & HIVEP3 & UTR3 & 0.405 & $63(15.7)$ & $94(17.5)$ & $28(19.6)$ & $\begin{array}{l}6.6 \mathrm{E}- \\
06\end{array}$ \\
\hline rs2810587 & 1 & 41973095 & $G>A$ & HIVEP3 & UTR3 & 0.400 & $64(15.7)$ & $94(17.6)$ & $27(19.4)$ & $\begin{array}{l}7.3 \mathrm{E}- \\
06\end{array}$ \\
\hline rs1383112 & 12 & 26287230 & $\mathrm{~T}>\mathrm{C}$ & & & 0.092 & $152(16.7)$ & $32(19.6)$ & $1(25.2)$ & $\begin{array}{l}8.4 \mathrm{E}- \\
06\end{array}$ \\
\hline rs1383113 & 12 & 26287240 & $A>G$ & & & 0.092 & $152(16.7)$ & $32(19.6)$ & $1(25.2)$ & $\begin{array}{l}8.4 \mathrm{E}- \\
06\end{array}$ \\
\hline rs2729628 & 12 & 26290639 & $\mathrm{~T}>\mathrm{C}$ & & & 0.092 & $152(16.7)$ & $32(19.6)$ & $1(25.2)$ & $\begin{array}{l}8.4 \mathrm{E}- \\
06\end{array}$ \\
\hline rs1780432 & 10 & 34184022 & $C>T$ & & & 0.208 & $112(18.2)$ & $69(15.9)$ & 4(13.3) & $\begin{array}{l}8.5 \mathrm{E}- \\
06\end{array}$ \\
\hline rs1740718 & 10 & 34184861 & $C>A$ & & & 0.208 & $112(18.2)$ & $69(15.9)$ & $4(13.3)$ & $\begin{array}{l}8.5 \mathrm{E}- \\
06\end{array}$ \\
\hline
\end{tabular}

Abbreviation: CHR (chromosome), BP (base pair), TL (telomere length) 


\begin{tabular}{|c|c|c|c|c|c|c|c|c|c|c|}
\hline SNP & CHR & BP & Alleles & Gene & Region & MAF & $\mathrm{DD}(\mathrm{TL})$ & $\operatorname{Dd}(T L)$ & $\mathrm{dd}(T L)$ & $\begin{array}{l}P \\
\text { value }\end{array}$ \\
\hline rs956613 & 10 & 34185869 & $\mathrm{G}>\mathrm{C}$ & & & 0.208 & 112(18.2) & 69(15.9) & $4(13.3)$ & $\begin{array}{l}8.5 \mathrm{E}- \\
06\end{array}$ \\
\hline rs74386538 & 22 & 27516762 & $A>G$ & & & 0.257 & 101(18.2) & 73(16.4) & 11(13.7) & $\begin{array}{l}8.6 \mathrm{E}- \\
06\end{array}$ \\
\hline rs417693 & 4 & 111211272 & $G>A$ & & & 0.124 & $140(16.5)$ & $44(19.2)$ & $1(24.4)$ & $\begin{array}{l}9.2 \mathrm{E}- \\
06\end{array}$ \\
\hline rs10915048 & 1 & 30682811 & $A>G$ & & & 0.254 & $99(16.1)$ & $78(18.4)$ & $8(20.0)$ & $\begin{array}{l}9.2 \mathrm{E}- \\
06\end{array}$ \\
\hline rs3809140 & 12 & 26278444 & $G>A$ & & & 0.089 & 153(16.7) & 31(19.6) & $1(25.2)$ & $\begin{array}{l}1.1 \mathrm{E}- \\
05\end{array}$ \\
\hline rs11243434 & 9 & 134448487 & $G>A$ & & & 0.097 & 153(16.7) & $28(19.5)$ & $4(22.3)$ & $\begin{array}{l}1.1 \mathrm{E}- \\
05\end{array}$ \\
\hline rs80083893 & 22 & 27516984 & $G>A$ & & & 0.265 & $99(18.2)$ & $74(16.4)$ & $12(13.9)$ & $\begin{array}{l}1.3 \mathrm{E}- \\
05\end{array}$ \\
\hline rs12788951 & 11 & 113471957 & $\mathrm{~T}>\mathrm{C}$ & & & 0.416 & $63(15.4)$ & $90(18.0)$ & $32(18.7)$ & $\begin{array}{l}1.5 \mathrm{E}- \\
05\end{array}$ \\
\hline rs7931613 & 11 & 82694032 & $\mathrm{~T}>\mathrm{C}$ & RAB30 & intron & 0.403 & $63(16.0)$ & $95(17.2)$ & $27(20.0)$ & $\begin{array}{l}1.5 \mathrm{E}- \\
05\end{array}$ \\
\hline
\end{tabular}

To investigate the effects of multiple SNPs on telomere length in our neuroblastoma patients, we performed a gene-based assay using the VEGAS2 algorithm ${ }^{28}$. We found that the CNTN4 gene had the most significant association $(P=1.0 \mathrm{E}-06)$. The results of the gene-based analysis are summarized in Supplementary Table 1.

\section{Discussion}

In this study, we investigated the association between telomere length in peripheral blood mononuclear cells, not tumor cells, and the clinical characteristics of tumors at diagnosis. Patients with an extra-abdominal primary tumor, lower stage tumor, MYCN non-amplified tumor, or low-risk tumor had longer germline telomere length than other patients. These clinical features are usually associated with better prognosis, and thus, it was unsurprising that longer telomere length was associated with better EFS and was an independent prognostic factor for EFS in our multivariate analysis. This is the first study to elucidate the clinical significance of germline telomere length in patients with neuroblastoma. We cannot explain the reason for the association between germline telomere length and the clinical characteristics of tumors. However, our results suggest that germline genomic characteristics, including telomere length, might affect the clinical characteristics of tumors at diagnosis and the treatment response.

For this reason, we performed genome-wide genotyping with a high-density bead chip to identify the genetic factors related to telomere length. In the GWAS analysis, novel risk SNPs, including HIVEP3 and LRRTM4, were significantly associated with telomere length (Table 3). The most significant association was with rs10842679 $(P=4.7 \mathrm{E}-07)$. That marker was adjacent to the BHLHE41 (basic helix-loop-helix family member e41) gene. BHLHE41 is known to be a transcription factor implicated in cellular functions such as proliferation, differentiation, and tumorigenesis ${ }^{27}$. It is possible that this marker is linked to genetic factors that directly or indirectly affect the expression of the BHLHE41 gene. Follow-up functional studies would be required to clarify the exact mechanism. Table 3 summarizes information about the genes with significant markers. Among them, many genes significant to the HIVEP3 gene were found. No direct functional association between neuroblastoma and the HIVEP3 gene has yet been reported. However, studies of other carcinomas have reported that they have higher levels of HIVEP3 and SOX9 messenger RNA expression than non-carcinoma cells ${ }^{29}$. In particular, patients with HIVEP3 and SOX9 overexpression 
showed a lower survival rate. In this study, patients with the recessive allele of the HIVEP3 gene had long telomere lengths. Follow-up studies comparing therapeutic effects and survival rates according to the expression of the HIVEP3 gene and telomere length changes are needed to clarify how telomere length is related to the function of the HIVEP3 gene.

A gene-based analysis was performed using the VEGAS2 algorithm, and the CNTN4 gene showed the most significant association ( $P=1.0 \mathrm{E}-06$; Supplementary Table 1). The rs71311728 marker within the CNTN4 gene also showed a significant association in the GWAS analysis $(P=0.0001)$. The rs71311728 marker within CNTN4 gene is a new marker with no reported association with other cancers. The CNTN4 gene encodes contactin 4, a member of the immunoglobulin superfamily ${ }^{30}$. The functions of these proteins are suggested to involve synaptic plasticity. In addition, they are known to be involved in axon growth, guidance, and fasciculation ${ }^{31}$. Although the details remain unclear, the effect of CNTN4 on the development of nerve endings and the development of neuroblastoma should be examined in future functional studies. To investigate the biological functions of the significant genes we identified, we used VEGAS2 to perform a gene ontology analysis. The results are summarized in Supplementary Table 2. The statistically significant GO categories (empirical $P<0.00005$ ) were cadherin binding, cell to cell pathway, cell leading edge, neurite morphogenesis, cell part morphogenesis, and neuron development.

This is the first GWAS study to investigate an association with telomere length in neuroblastoma patients, and we found that the CNTN4 gene is associated with changes in telomere length in Korean neuroblastoma patients. Our number of samples was insufficient due to the rarity of neuroblastoma. However, the new genes and markers discovered through this study will contribute to other GWAS studies that can be conducted in various ethnicities in the future.

In conclusion, we found that longer germline telomere length is associated with favorable prognostic factors at diagnosis and eventually a better EFS in patients with neuroblastoma. In addition, the GWAS demonstrated that genetic markers and genes related to telomere length were associated with neurite morphogenesis and neuron development in neuroblastoma. A further study with a larger cohort of patients and functional studies are needed.

\section{Declarations}

\section{Acknowledgement}

This work was supported by a grant from the National Research Foundation of Korea (NRF), which is funded by the Korean government (NRF-2019R1F1A1041365). The samples used in this study were obtained from the Samsung Medical Center Biobank.

\section{Competing Interest}

The authors declare that they have no competing interests.

\section{Data availability}

All data analyzed or generated during this study are included in the article.

\section{References}

1. Blackburn, E. H. Switching and signaling at the telomere. Cell 106, 661-673. http://doi.org/10.1016/s00928674(01)00492-5 (2001).

2. Palm, W. \& de Lange, T. How shelterin protects mammalian telomeres. Annu Rev Genet 42, 301-334. http://doi.org/10.1146/annurev.genet.41.110306.130350 (2008). 
3. Siderakis, M. \& Tarsounas, M. Telomere regulation and function during meiosis. Chromosome Res 15, 667-679. http://doi.org/10.1007/s10577-007-1149-7 (2007).

4. Autexier, C. \& Lue, N. F. The structure and function of telomerase reverse transcriptase. Annu Rev Biochem 75, $493-517$. http://doi.org/10.1146/annurev.biochem.75.103004.142412 (2006).

5. Njajou, O. T. et al. Telomere length is paternally inherited and is associated with parental lifespan. Proc Natl Acad Sci U S A 104, 12135-12139. http://doi.org/10.1073/pnas.0702703104 (2007).

6. Bojesen, S. E. Telomeres and human health. J Intern Med 274, 399-413. http://doi.org/10.1111/joim.12083 (2013).

7. Herrmann, M., Pusceddu, I., Marz, W. \& Herrmann, W. Telomere biology and age-related diseases. Clin Chem Lab Med 56, 1210-1222. http://doi.org/10.1515/cclm-2017-0870 (2018).

8. Maciejowski, J. \& de Lange, T. Telomeres in cancer: tumour suppression and genome instability. Nat Rev Mol Cell Biol 18, 175-186. http://doi.org/10.1038/nrm.2016.171 (2017).

9. Patel, C. J., Manrai, A. K., Corona, E. \& Kohane, I. S. Systematic correlation of environmental exposure and physiological and self-reported behaviour factors with leukocyte telomere length. Int J Epidemiol 46, 44-56. http://doi.org/10.1093/ije/dyw043 (2017).

10. Codd, V. et al. Identification of seven loci affecting mean telomere length and their association with disease. Nat Genet 45, 422-427, 427e421-422. http://doi.org/10.1038/ng.2528 (2013).

11. Frias, C. et al. Telomere shortening is associated with poor prognosis and telomerase activity correlates with DNA repair impairment in non-small cell lung cancer. Lung Cancer 60, 416-425. http://doi.org/10.1016/j.lungcan.2007.11.001 (2008).

12. D'Mello, M. J. et al. Association between shortened leukocyte telomere length and cardiometabolic outcomes: systematic review and meta-analysis. Circ Cardiovasc Genet 8, 82-90. http://doi.org/10.1161/CIRCGENETICS.113.000485 (2015).

13. Jang, J. S. et al. Telomere length and the risk of lung cancer. Cancer Sci 99, 1385-1389. http://doi.org/10.1111/j.13497006.2008.00831.x (2008).

14. Shen, M. et al. A prospective study of telomere length measured by monochrome multiplex quantitative PCR and risk of lung cancer. Lung Cancer 73, 133-137. http://doi.org/10.1016/j.lungcan.2010.11.009 (2011).

15. Ma, H. et al. Shortened telomere length is associated with increased risk of cancer: a meta-analysis. PLoS One 6, e20466. http://doi.org/10.1371/journal.pone.0020466 (2011).

16. Karimi, B., Yunesian, M., Nabizadeh, R., Mehdipour, P. \& Aghaie, A. Is Leukocyte Telomere Length Related with Lung Cancer Risk?: A Meta-Analysis. Iran Biomed J 21, 142-153. http://doi.org/10.18869/acadpub.ibj.21.3.142 (2017).

17. Shen, J. et al. Telomere length, oxidative damage, antioxidants and breast cancer risk. Int J Cancer 124, 1637-1643. http://doi.org/10.1002/ijc.24105 (2009).

18. Sommerfeld, H. J. et al. Telomerase activity: a prevalent marker of malignant human prostate tissue. Cancer Res 56, 218-222 (1996).

19. Artandi, S. E. et al. Constitutive telomerase expression promotes mammary carcinomas in aging mice. Proc Natl Acad Sci U S A 99, 8191-8196. http://doi.org/10.1073/pnas.112515399 (2002).

20. Blasco, M. A. et al. Telomere shortening and tumor formation by mouse cells lacking telomerase RNA. Cell 91, 25-34. http://doi.org/10.1016/s0092-8674(01)80006-4 (1997).

21. Babizhayev, M. A., Savel'yeva, E. L., Moskvina, S. N. \& Yegorov, Y. E. Telomere length is a biomarker of cumulative oxidative stress, biologic age, and an independent predictor of survival and therapeutic treatment requirement associated with smoking behavior. Am J Ther 18, e209-226. http://doi.org/10.1097/MJT.0b013e3181cf8ebb (2011).

22. Seow, W. J. et al. Telomere length in white blood cell DNA and lung cancer: a pooled analysis of three prospective cohorts. Cancer Res 74, 4090-4098. http://doi.org/10.1158/0008-5472.CAN-14-0459 (2014).

23. Sanchez-Espiridion, B. et al. Telomere length in peripheral blood leukocytes and lung cancer risk: a large case-control study in Caucasians. Cancer Res 74, 2476-2486. http://doi.org/10.1158/0008-5472.CAN-13-2968 (2014). 
24. Gramatges, M. M., Telli, M. L., Balise, R. \& Ford, J. M. Longer relative telomere length in blood from women with sporadic and familial breast cancer compared with healthy controls. Cancer Epidemiol Biomarkers Prev 19, 605-613.

http://doi.org/10.1158/1055-9965.EPI-09-0896 (2010).

25. Zheng, Y. L. et al. Telomere length in blood cells and breast cancer risk: investigations in two case-control studies. Breast Cancer Res Treat 120, 769-775. http://doi.org/10.1007/s10549-009-0440-z (2010).

26. Cawthon, R. M. Telomere measurement by quantitative PCR. Nucleic Acids Res 30 , e47.

http://doi.org/10.1093/nar/30.10.e47 (2002).

27. Sun, Y. et al. Loss of the basic helix-loop-helix transcription factor Bhlhe41 induces cell death and impairs neurite outgrowth in Neuro2a cells. Mol Cell Biochem 450, 167-174. http://doi.org/10.1007/s11010-018-3383-z (2019).

28. Mishra, A. \& Macgregor, S. VEGAS2: Software for More Flexible Gene-Based Testing. Twin Res Hum Genet 18, 86-91. http://doi.org/10.1017/thg.2014.79 (2015).

29. Qin, G. Q. et al. Combined overexpression of HIVEP3 and SOX9 predicts unfavorable biochemical recurrence-free survival in patients with prostate cancer. Onco Targets Ther 7, 137-146. http://doi.org/10.2147/OTT.S55432 (2014).

30. Hansford, L. M. et al. Cloning and characterization of the human neural cell adhesion molecule, CNTN4 (alias BIG-2). Cytogenet Genome Res 101, 17-23. http://doi.org/10.1159/000073412 (2003).

31. Yoshihara, Y. et al. Overlapping and differential expression of BIG-2, BIG-1, TAG-1, and F3: four members of an axonassociated cell adhesion molecule subgroup of the immunoglobulin superfamily. J Neurobiol 28, 51-69. http://doi.org/10.1002/neu.480280106 (1995).

\section{Figures}

Figure 1.

(A)

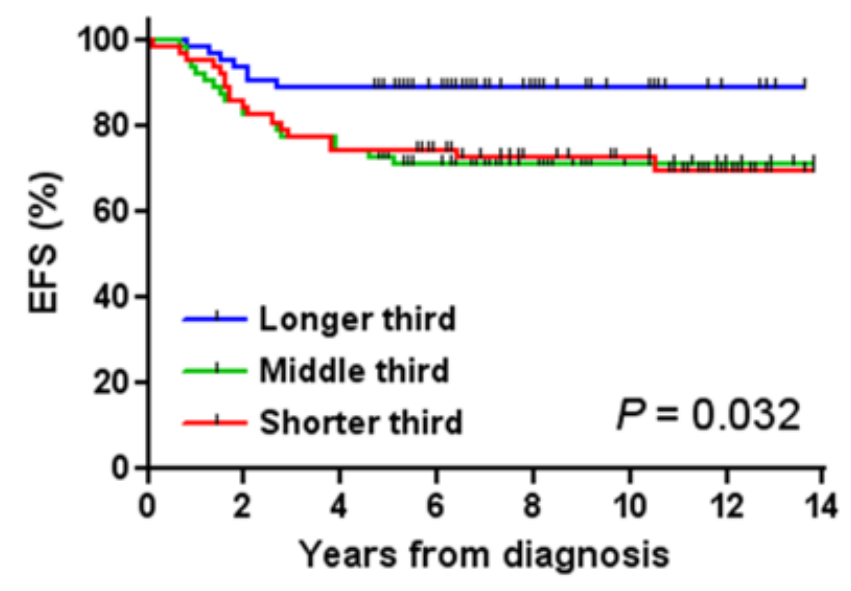

(B)

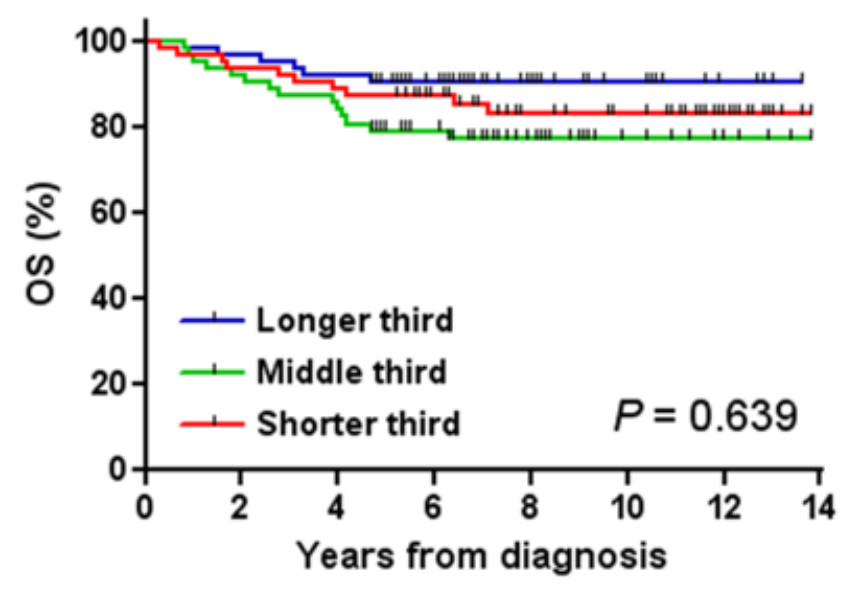

\section{Figure 1}

Survival data according to telomere length. (A) Patients with longer third telomeres showed longer event-free survival than other patients, (B) but there was no statistical difference in overall survival. 
Figure 2.

(A)

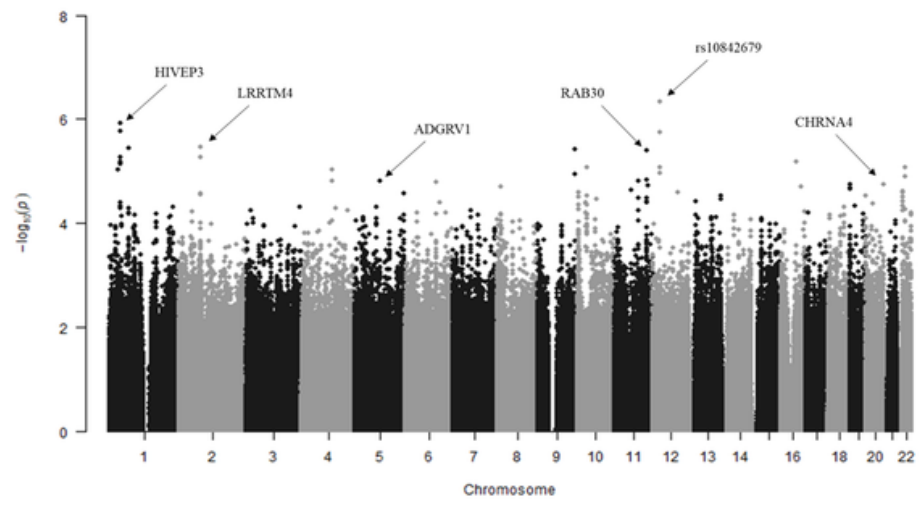

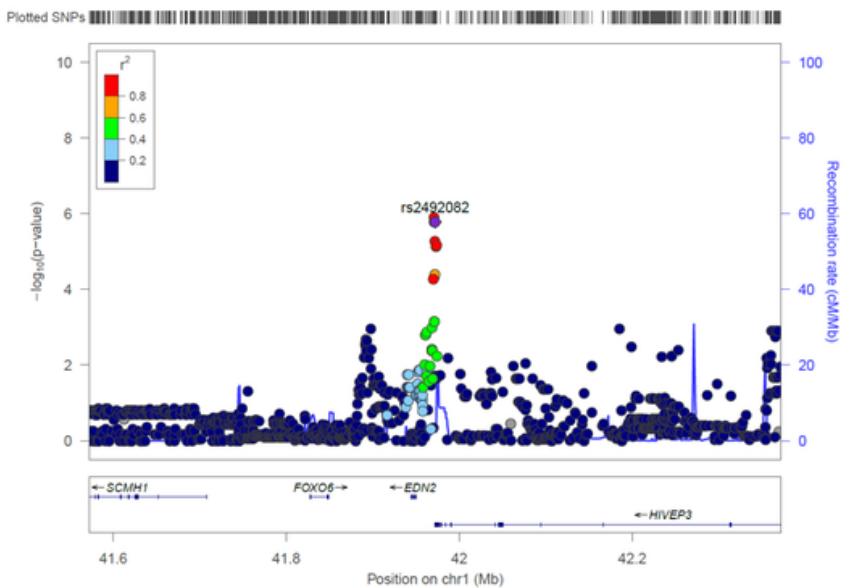

Figure 2

Manhattan plot and regional association plot. (A) $P$ values from the genome-wide association study. The Manhattan plot shows the $P$ values for the risk of neuroblastoma calculated using a logistic regression analysis. The $\mathrm{X}$-axis represents the single nucleotide polymorphism (SNP) markers on each chromosome. The highest $P$ value $(P=4.7 \mathrm{E}-07)$ was observed for rs10842679 on 12p.12.1. (B) Regional association plots at CNTN4. Regional association plots containing both genotypes and SNPs within $400 \mathrm{~kb}$ of $C N T N 4$ were generated by LocusZoom. The significance of the association (- $\log 10$-transformed $\mathrm{p}$ values) and recombination rate are plotted. SNPs are colored to reflect pairwise linkage disequilibrium $\left(r^{2}\right)$ with the most significantly associated genotyped SNPs in the 1000 Genomes Project Phase 1 interim release Asian (ASN) population genotypes. The most significant genotyped SNPs are labeled and shown in purple.

\section{Supplementary Files}

This is a list of supplementary files associated with this preprint. Click to download.

- manuscripttelomereNBSNP20210910SupplementaryTables.docx 